\title{
SIRT6 promotes the Warburg effect of papillary thyroid cancer cell BCPAP through reactive oxygen species
}

This article was published in the following Dove Press journal: OncoTargets and Therapy

\section{Weiping Yu* \\ Zhou Yang* \\ Renhong Huang \\ Zhijun Min \\ Min Ye}

Department of General Surgery, Shanghai Pudong Hospital, Fudan University Pudong Medical Center, Shanghai 201399, People's Republic of China

*These authors contributed equally to this work
Correspondence: Min Ye

Department of General Surgery, Shanghai Pudong Hospital, Fudan University Pudong Medical Center, 2800 Gongwei Road, Huinan Town, Pudong, Shanghai 201399, People's Republic of China Email yemincool@I26.com
Purpose: Our previous study demonstrated that SIRT6 is upregulated in papillary thyroid cancer (PTC) and enhances tumor aggressiveness. In this study, we further researched its influence in the Warburg effect.

Methods: SIRT6-upregulated and downregulated BCPAP cells and negative control BCPAP-NC groups were generated with lentiviral vectors. In these two cell lines, reactive oxygen species (ROS) were detected by dichlorodihydrofluorescein diacetate. Expression of the key Warburg effect genes including GLUT1, HK2, GAPDH, PGK1, ENO1, PKM2 and LDHA was measured by quantitative real-time PCR and western blotting. Glucose uptake, lactate production and the ATP content of cells were detected with assay kits. The ROS scavenger $N$-acetylcysteine was used for treatment of BCPAP-SIRT6, and the same measurements as described above were detected again.

Results: Compared with the BCPAP-NC group, expression of the key Warburg effect genes including Glut1, HK2 and GAPDH and their protein products was upregulated in the BCPAP-SIRT6 group, whereas BCPAP-shSIRT6 showed significant downregulation. Meanwhile, ROS, glucose uptake, lactate production and ATP content of the BCPAPSIRT6 group were also significantly increased, and BCPAP-shSIRT6 showed significant downregulation. Furthermore, upregulation of key Warburg effect genes and glucose uptake, lactate production and ATP content were all rescued after treatment with ROS scavenger. Conclusion: SIRT6 promoted the Warburg effect of PTC cells via upregulation of ROS. Inhibition of ROS in SIRT6-upregulated cells could rescue activation of the Warburg effect. Keywords: sirtuin 6, Warburg effect, reactive oxygen species, papillary thyroid cancer

\section{Introduction}

Sirtuin 6 (SIRT6), astress responsive protein deacetylase and mono-ADP ribosyltransferase enzyme, is encoded by the SIRT6 gene and is associated with aging, DNA repair, telomere maintenance, glycolysis, inflammation, etc, via multiple molecular pathways. In cancer research, SIRT6 was also found to play arole as an oncogene or anti-oncogene in different tumors. These research studies focused on different pathways regulating tumorigenesis and tumor progress. ${ }^{1,2}$ Our previous study demonstrated that SIRT6 is upregulated in papillary thyroid cancer (PTC) and enhances tumor aggressiveness via the BRAF/ERK/Mcl-1 pathway. ${ }^{3}$ We found that silencing of SIRT6 weakens PTC cells, colony formation, migration and invasion, and blocked the cell cycle. In this study, we innovatively looked into the relationship between SIRT6 and the Warburg effect, and explored its possible specific mechanisms. 
Malignant tumor is not only agenetic disease, but also an energy metabolic disease. Even when the oxygen supply is sufficient, tumor cells obtain energy mainly by glycolysis, which is also called aerobic glycolysis or the Warburg effect. This abnormal glycolysis of tumor cells promotes glucose uptake and lactate production, which is conducive to tumorigenesis and tumor progress. The coding genes of key glycolysis enzymes are directly responsible for the regulation of the Warburg effect, including GLUT1, HK2, GAPDH, PGK1, ENO1, PKM2 and LDHA. ${ }^{4}$ Furthermore, transcription factors play direct and key roles in regulating these enzyme genes, such as hypoxia-inducible factor-1 (hif-1), cMYC, NF-kb and p53. Previous studies of SIRT6 and the Warburg effect were also concentrated on transcription factors. In embryonic stem cells and mouse embryonic fibroblasts, SIRT6-deficient cells exhibit increased Hif- $1 \alpha$ activity and show increased glucose uptake with upregulation of glycolysis and diminished mitochondrial respiration. ${ }^{55}$ In another study, SIRT6 also took arole as anovel regulator of ribosome metabolism by co-repressing MYC transcriptional activity. ${ }^{6}$ In fact, factors regulating the Warburg effect are varied, not limited to transcription factors. Reactive oxygen species (ROS) also play an important role in this effect. ROS are aclass of oxygen atoms or clusters formed by oxygen that contain oxygen in their molecular composition, as well as having more active chemical properties than oxygen itself. In glioblastoma, deficiency of PINK1 inhibited the Warburg effect through ROS-dependent stabilization of HIF-1 $\alpha$. Therefore, we hypothesized whether SIRT6 was capable of influencing the Warburg effect via ROS.

\section{Materials and methods}

\section{Cell line and cell culture}

Human PTC cell line BCPAP was purchased from the University of Colorado Cancer Center Cell Bank. All cells were cultured in RPMI 1640 medium supplemented with 10\% FBS (Thermo Fisher Scientific, Waltham, MA, USA) in an atmosphere of $5 \% \mathrm{CO}_{2}$ and $37{ }^{\circ} \mathrm{C}$.

\section{Lentiviral vector and infection}

The cDNA of human SIRT6 was purchased from Origene (RC202833; Rockville, MD, USA) and shRNA of SIRT6 (sequence: GCAGTCTTCC AGTGTGGTGTT) were cloned into the pCDH-CMV-
MCS-EF1-Puro lentiviral vector for the pCDH-SIRT6 and pCDH-shSIRT6 plasmids. According to the instructions of Lipofectamine 3000, the pCDH-SIRT6/shSIRT6 plasmid, psPAX2 and PMG.2G were co-transfected into HEK293T cells to obtain overexpressing SIRT6 lentivirus. Then, the BCPAP cell was infected by lentivirus (multiplicity of infection=25) and screened by puromycin $(5 \mu \mathrm{g} / \mathrm{mL}, 72 \mathrm{~h})$ to generate the stably SIRT6-upregulated cell BCPAP-SIRT6 and the SIRT6downregulated cell BAPAP-shSIRT6. Meanwhile, negative control constructed with empty vector was also generated as BCPAP-NC. The expression of SIRT6 was confirmed by western blotting and quantitative real-time PCR (qRT-PCR) analysis.

\section{RNA extraction and qRT-PCR}

Total RNA from PTC cells in each group was extracted by Trizol Reagent (Thermo Fisher Scientific). cDNA was obtained from total RNA using the PrimeScript ${ }^{\mathrm{TM}}$ RT reagent kit (Takara Bio, Inc., Otsu, Japan). The expression of mRNA was assessed by qRT-PCR, which was carried out in triplicate by an SYBR Premix Ex Taq ${ }^{\mathrm{TM}}$ kit (Takara Bio, Inc.) and an ABI 7900HT Real-Time PCR system (Thermo Fisher Scientific). The primers used are presented in Table 1. $\beta$-actin was used to normalize the results of qRT-PCR and the comparative cycle threshold values $\left(2^{-}\right.$ $\Delta \Delta \mathrm{Ct}$ ) were adopted to analyze the final results.

\section{Protein extraction and western blotting analysis}

The total cellular proteins were extracted using RIPA lysis buffer with $1 \%$ phenylmethanesulfonyl fluoride. Then, equal amounts $(20 \mu \mathrm{g})$ of protein determined by the BCA protein assay kit (Thermo Fisher Scientific) were separated using $10 \%$ SDS-PAGE gels. The proteins were then transferred to polyvinylidene difluoride membranes $(0.45 \mathrm{~mm}$; Solarbio, Beijing, People's Republic of China). The membranes were blocked with $5 \%$ nonfat milk for 1 hat room temperature and then incubated with primary antibodies at $4{ }^{\circ} \mathrm{C}$ for $12 \mathrm{~h}$. The following antibodies were tested: SIRT6, Glut1, c-MYC and p53 rabbit polyclonal antibodies (1:1000; Proteintech, USA). $\beta$-actin rabbit polyclonal antibodies (1:4000; Proteintech) were used as loading controls and for normalization. The secondary antibodies were anti-rabbit antibodies and were conjugated to horseradish peroxidase (1:4000; Proteintech). The antibodies were used at a1:4000 dilution and were incubated for approximately 1 hat room temperature. The bands 
Table I The primers for quantitative real-time PCR

\begin{tabular}{|l|l|l|}
\hline Gene & Forward primer & Reverse primer \\
\hline SIRT6 & GCACCGTGGCTAAGGCAAGG & GTGATGGACAGGTCGGCGTTC \\
Actin & GGGACCTGACTGACTACCTC & TCATACTCCTGCTTGCTGAT \\
PKM & ACTGGCATCATCTGTACCATTG & AGCCACATTCATTCCAGACTTA \\
LDHA & GGTTGGTGCTGTTGGCATGG & TGCCCCAGCCGTGATAATGA \\
HK2 & CGACAGCATCATTGTTAAGGAG & GCAGGAAAGACACATCACATTT \\
GlutI & CTGGCATCAACGCTGTCTTC & GCCTATGAGGTGCAGGGTC \\
Pnol & GCCTCCTGCTCAAAGTCAAC & AACGATGAGACACCATGACG \\
GAPDH & TTCTGTTCTTGAAGGACTGTGT & CTTTAACCTTGTTCCCAGAAGC \\
\hline
\end{tabular}

were visualized with ECL reagents (Thermo Fisher Scientific) and developed by Omega Lum G(Aplegen, USA).

\section{Glucose uptake, lactate production and ATP content assays}

For glucose uptake and lactate production assays, $5 \times 10^{5}$ cells were seeded onto 6-well plates and cultured at $37^{\circ} \mathrm{C}$. After $24 \mathrm{~h}, 5 \mu \mathrm{L}$ cell culture supernatant was collected in 96-well plates and mixed with $200 \mu \mathrm{L}$ Glucose (HK) Assay Reagent (Sigma-Aldrich Co., St.Louis, USA) or $100 \mu \mathrm{L}$ Lactate Assay Reagent (Sigma-Aldrich). After 20 min of incubation at $37{ }^{\circ} \mathrm{C}$, the absorbance at $340 \mathrm{~nm}$ $\left(\mathrm{OD}_{340}\right)$ was determined with amicroplate reader. At the same time, astandard curve was established through glucose and lactate standards. $\mathrm{OD}_{340}$ was substituted into the standard curve to get the glucose or lactate level. For the ATP content assay, $2 \times 10^{3}$ cells were seeded onto $96-$ well plates and incubated for 48 h.Culture medium was removed and the ATPLite luminescent assay (PerkinElmer Inc., Waltham, MA, USA) was added. After $30 \mathrm{~min}$ of incubation at $37^{\circ} \mathrm{C}$, luminescence was monitored. At the same time, the standard curve was established through ATP standard. The luminescence intensity was substituted into the standard curve to get the ATP level. Glucose uptake, lactate production and ATP content were all normalized by cell number.

\section{ROS detection and inhibition}

For ROS detection, $5 \times 10^{5}$ cells were seeded onto 6-well plates and cultured at $37{ }^{\circ} \mathrm{C}$ for $24 \mathrm{~h}$. Then, $1 \mathrm{~mL}$ FBSfree medium diluted dichlorodihydrofluorescein diacetate (DCFH-DA; Beyotime, Shanghai, People's Republic of China) was added to the dish after removing old culture medium. After incubation for $20 \mathrm{~min}$ at $37{ }^{\circ} \mathrm{C}$, cells were washed with FBS-free medium 3 times. Then, the fluorescence intensity of cells was detected with amicroplate reader with $488 \mathrm{~nm}$ excitation wavelength and $525 \mathrm{~nm}$ emission wavelength. For ROS inhibition, $5 \mathrm{mM} N$-acetylcysteine (NAC) was added in culture medium for $2 \mathrm{~h}$. The inhibition of ROS was confirmed with DCFH-DA.

\section{Statistical analysis}

SPSS software (version 19.0; IBM Corporation, Armonk, NY, USA) was used for statistical analysis of all experimental data. GraphPad Prism (version 7; GraphPad Software Inc., La Jolla, CA, USA) was used to determine the statistical results. All data are expressed as the mean \pm SD. The statistical analysis of the data from 2 groups was performed using a two-tailed Student's $t$-test. The comparisons of multiple groups were performed by one-way ANOVA and then aleast significant difference $t$-test. $P<0.05$ was considered to be significant.

\section{Results}

\section{SIRT6 showed positive correlation with key Warburg effect genes}

We studied co-expression between SIRT6 and key Warburg effect genes through GEPIA online tools based on The Cancer Genome Atlas database (http://gepia.can cer-pku.cn/). $P \square 0.05$ was thought statistically significant. We found that Eno1, GAPDH and PKM showed apositive correlation with SIRT6 in PTC patients. Inside, GAPDH showed high co-expression for which $P=7.0^{-12}$ and $\mathrm{R}=0.3$ (Figure 1).

\section{SIRT6 upregulated key Warburg effect genes}

Stable SIRT6-upregulated and downregulated PTC cell lines BCPAP-SIRT6 and BCPAP-shSIRT6 were 

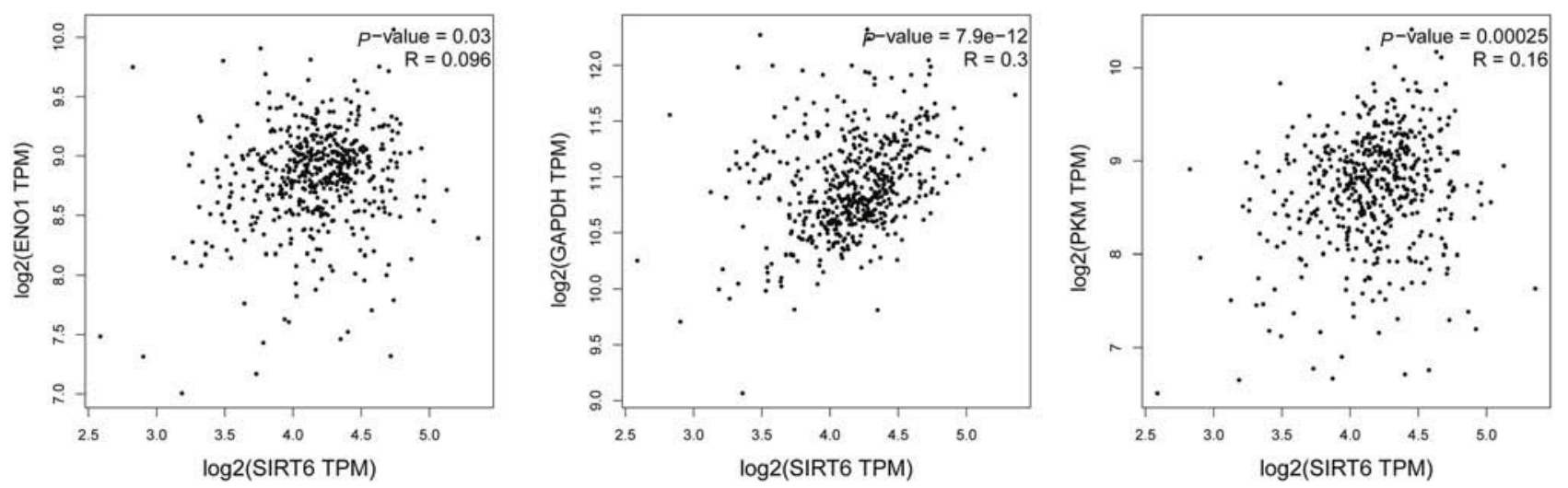

Figure I Co-expression between SIRT6 and key Warburg effect genes.

successfully generated and confirmed with western blotting and qRT-PCR. Both mRNA expression (Figure 2B) and protein expression (Figure 2A) of SIRT6 in the BCPAP-SIRT6 group were significantly upregulated compared with the BCPAP-NC group, and BCPAPshSIRT6 showed significant downregulation. Interestingly, mRNA expression of key Warburg effect genes PKM, Glut1, HK2, LDHA, Eno1, PGK1 and GAPDH (Figure 2D) and protein expression of Glut1 (Figure 2C) were also significantly increased in the BCPAP-SIRT6 group compared with the BCPAP-NC group. Meanwhile, these genes were significantly downregulated in the BCPAP-shSIRT6 group.

\section{SIRT6 increased glucose uptake, lactate production and ATP content of cells}

We further detected glucose uptake (Figure 3A) and lactate production (Figure $3 \mathrm{~B}$ ) from cell culture medium, and found an obvious increase in the BCPAP-SIRT6 group compared with the BCPAP-NC group. Then, the ATP content of the two cell lines was measured by ATPLite luminescent assay (Figure 3C). The same trend of higher ATP content was detected in the BCPAP-SIRT6 group as in the BCPAP-NC group. All data above in the BCPAPSIRT6 group were statistically significant. All of these measurements in BCPAP-shSIRT6 showed significant downregulation compared with BCPAP-NC.
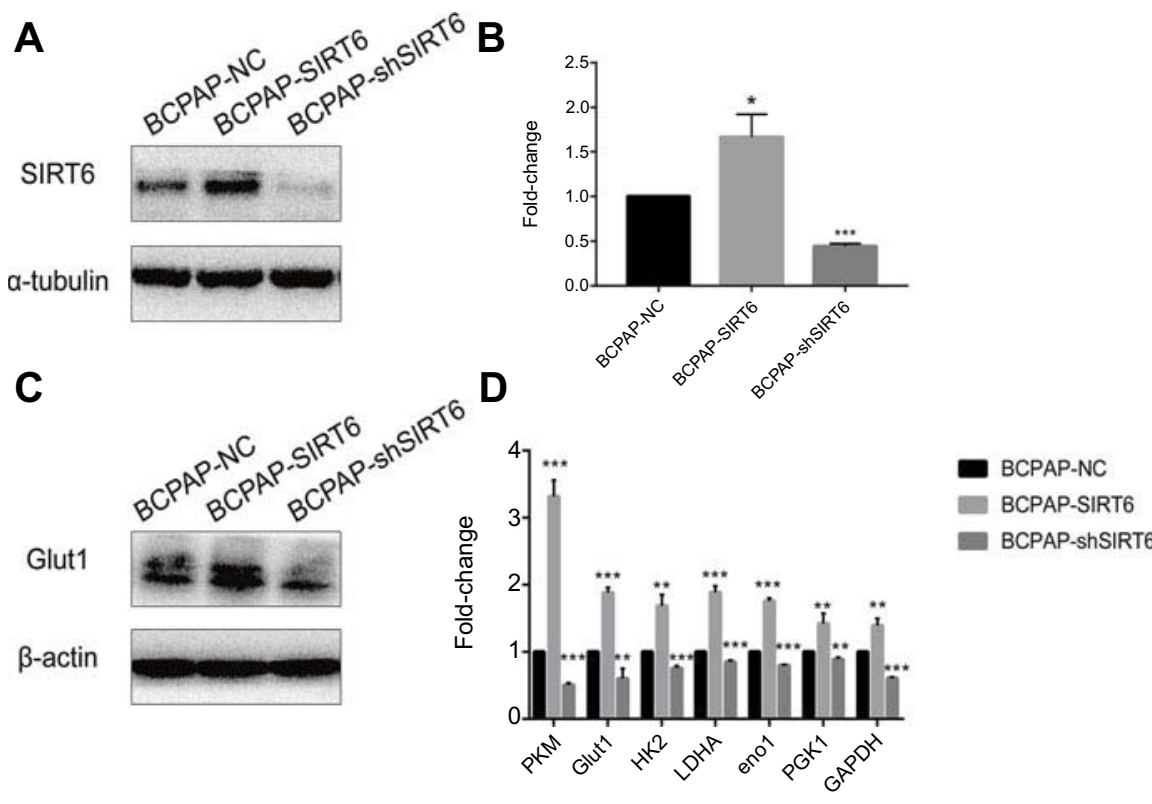

Figure 2 (A) Protein expression of SIRT6 in BCPAP cells was detected by western blotting. (B) mRNA expression of SIRT6 in BCPAP cells was detected by quantitative real-time PCR, and the results were analyzed. (C) Glutl protein expression in BCPAP cells was detected by western blotting. (D) Key Warburg effect gene expression in BCPAP cells was analyzed.

Notes: All group values are given as mean \pm SD. Compared with BCPAP-NC: $* P<0.05, * * P<0.01$ and $* * * P<0.001$. 
A

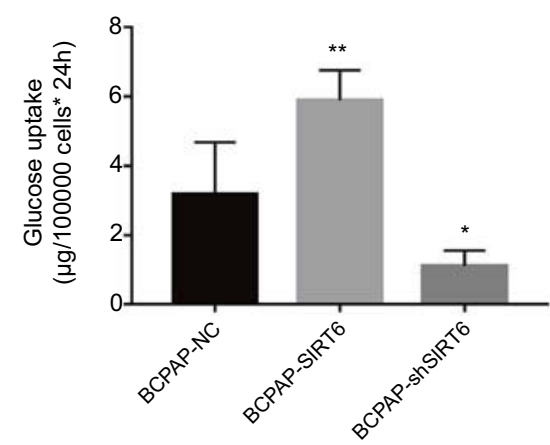

B

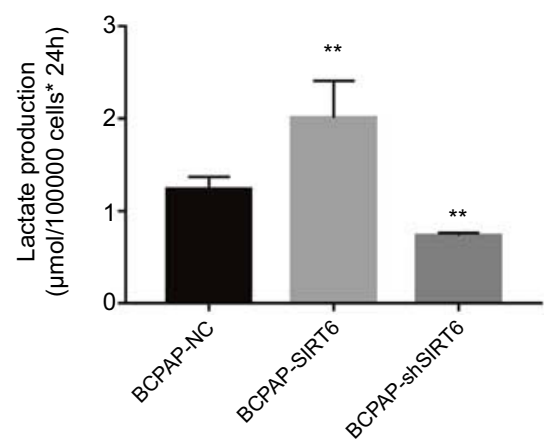

C

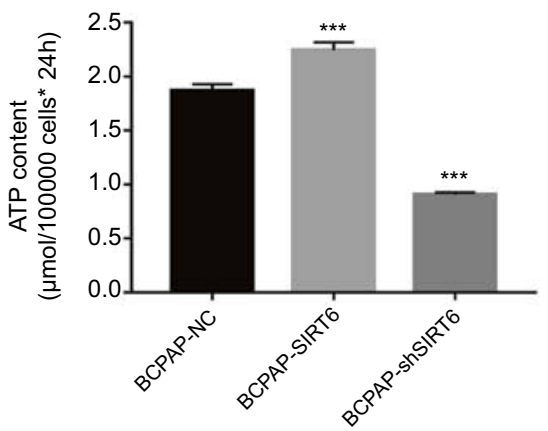

Figure 3 (A) Glucose uptake of BCPAP cells was detected and analyzed from cell culture medium. (B) Lactate production of BCPAP cells was detected and analyzed from cell culture medium. (C) ATP content of BCPAP cells was detected and analyzed by ATPLite luminescent assay. Notes: All group values are given as mean \pm SD. Compared with BCPAP-NC: $* P<0.05, * * P<0.01$ and $* * * P<0.001$.

\section{SIRT6 increased ROS production, whereas no effect on expression of c- MYC or $\mathrm{p} 53$}

c-MYC and p53 protein expression was detected via western blotting. However, no statistical significance was found between BCPAP-SIRT6 and BCPAP-NC (Figure 4A). We further measured ROS production through DCFH-DA, and found asignificant upregulation in the BCPAP-SIRT6 group compared with the BCPAP-NC group (Figure 4B).

\section{Inhibition of ROS rescued expression of key Warburg effect genes of BCPAP- SIRT6}

ROS scavenger NAC (5mM) was added in BCPAP-SIRT6 cell culture medium for $2 \mathrm{~h}$, and we found an obvious inhibition of ROS production (Figure 5A). Then, qRT-PCR (Figure 5C) and western blotting (Figure 5B) were performed again for detecting expression of key Warburg effect genes. Interestingly, increased mRNA expression of PKM, Glut1,
A

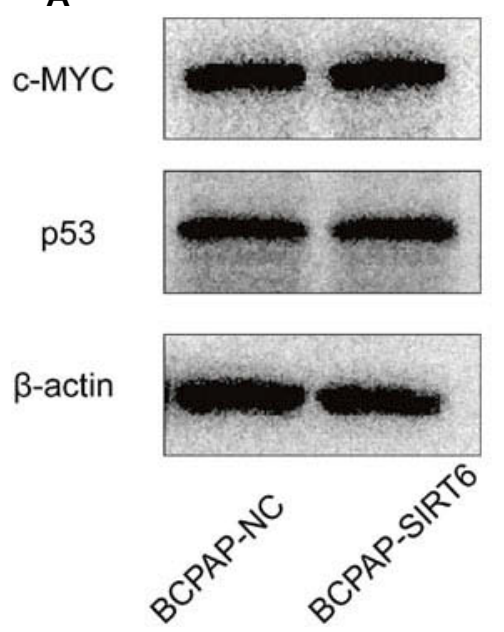

B

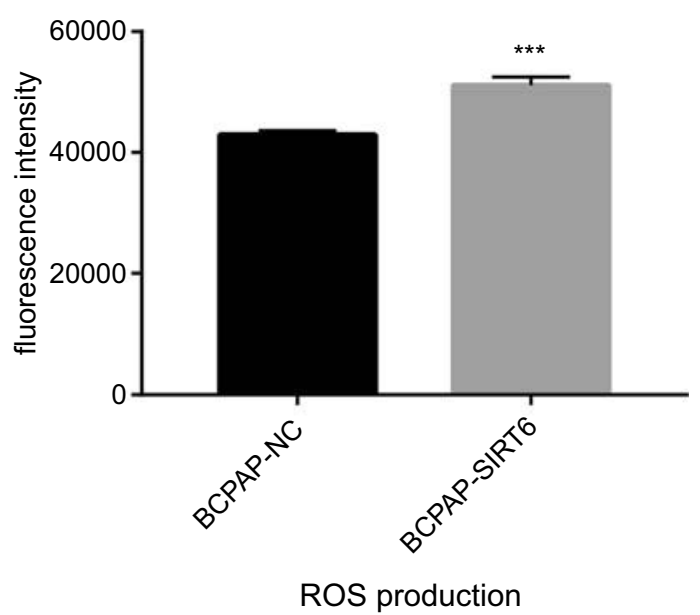

Figure 4 (A) c-MYC and p53 protein expression was detected by western blotting. (B) ROS production measured by dichlorodihydrofluorescein diacetate, and the result was analyzed.

Notes: The value is given as mean \pm SD. $* * * P<0.001$.

Abbreviation: ROS, reactive oxygen species. 
A

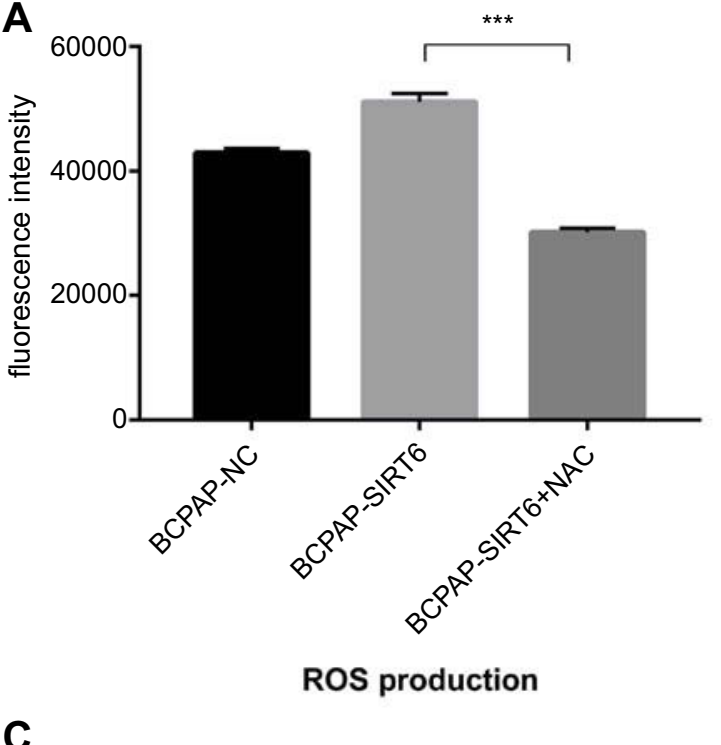

B

Glut1

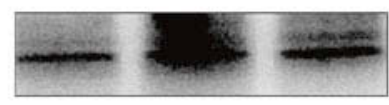

$\beta$-actin
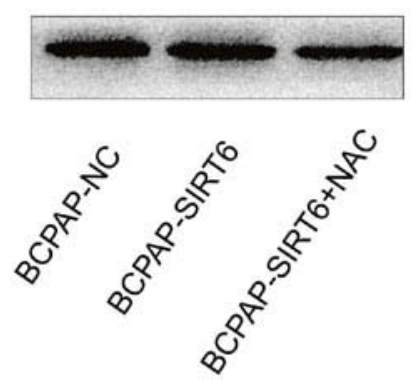

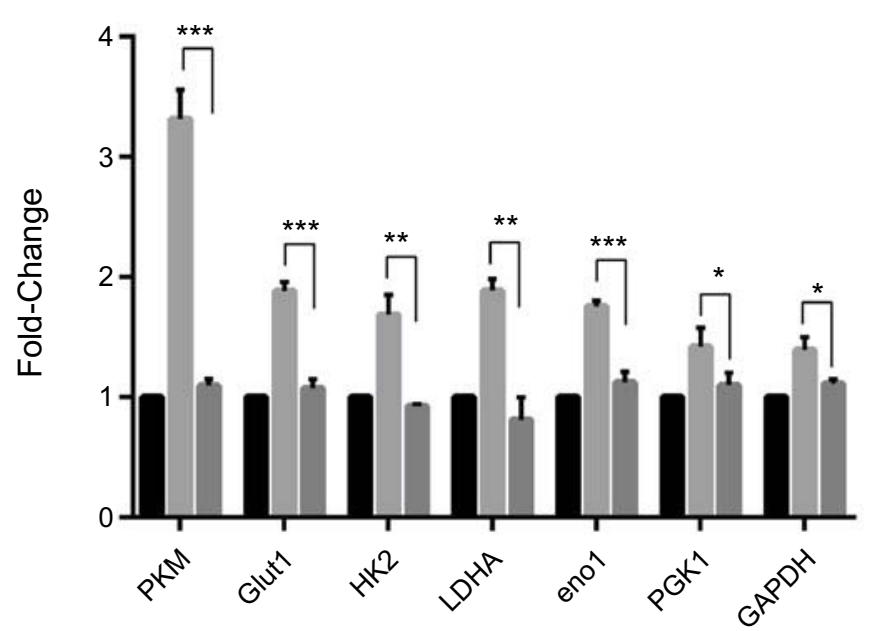

BCPAP-NC

BCPAP-SIRT6

BCPAP-SIRT6+NAC

Figure 5 (A) NAC could obviously inhibit ROS in BCPAP-SIRT6. (B) protein expression of Glutl was rescued in BCPAP-SIRT6. (C) mRNA expression of PKM, Glut I, HK2, LDHA, Enol, PGKI and GAPDH was rescued in the BCPAP-SIRT6 group.

Notes: All group values are given as mean $\pm S D$. $* P<0.05$, $* * P<0.01$ and $* * * P<0.001$.

Abbreviations: NAC, $\mathrm{N}$-acetylcysteine; ROS, reactive oxygen species.

HK2, LDHA, Eno1, PGK1 and GAPDH and protein expression of Glut1 were all significantly rescued in BCPAP-SIRT6.

Inhibition of ROS rescued glucose uptake, lactate production and ATP content of BCPAP-SIRT6

After inhibition of ROS in the BCPAP-SIRT6 group via NAC as described above, glucose uptake (Figure 6A), lactate production (Figure 6B) and ATP content (Figure 6C) were measured again using the methods described above. Increased glucose uptake, lactate production and ATP content of BCPAP-SIRT6 fell back to the original level.

\section{Discussion}

The Warburg effect is an important metabolic foundation of cancer cell proliferation, cell progress, metastasis and drug resistance. It also occurs and performs as ahigh level of glucose uptake, lactate production and ATP content in PTC. ${ }^{7}$ Meanwhile, glycolysis key enzyme genes GLUT1, HK2, GAPDH, PGK1, Eno1, PKM2 and LDHA are also directly responsible for this effect. Aprevious study confirmed that Glut1 was upregulated in PTC, and was associated with increased mortality. ${ }^{8}$ In this research, we successfully generated stable SIRT6-upregulated and downregulated PTC cell lines BCPAP-SIRT6 and BCPAP-shSIRT6. We also found that mRNA expression 

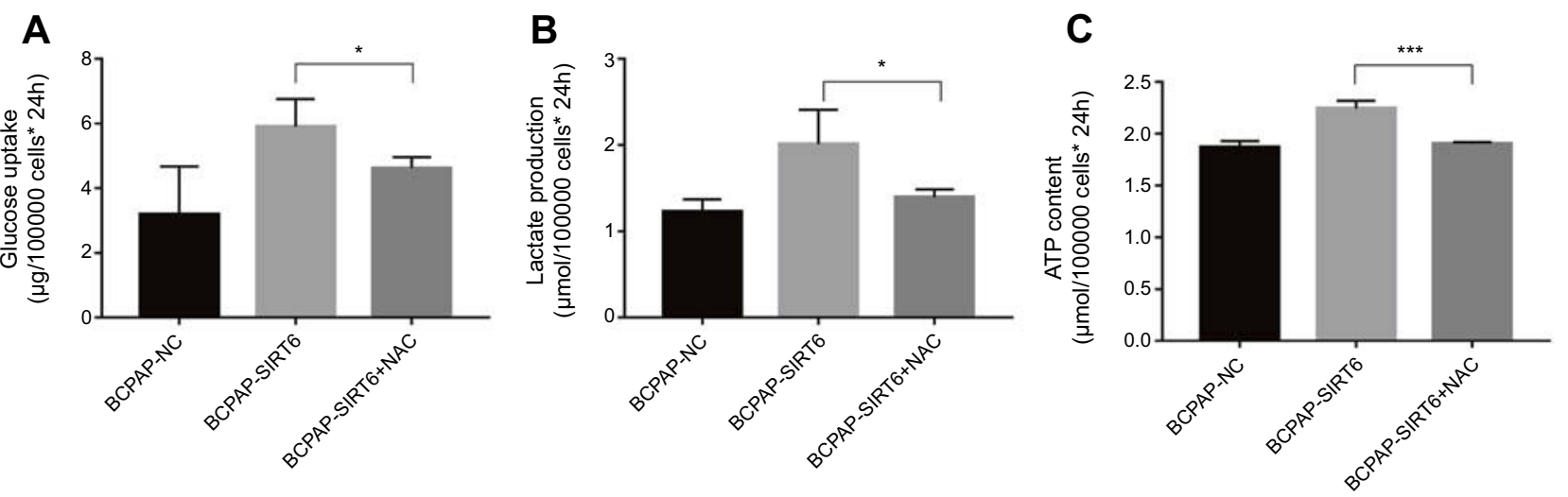

Figure 6 (A) Glucose uptake was detected and analyzed from cell culture medium. (B) Lactate production was detected and analyzed from cell culture medium. (C) ATP content was detected and analyzed by ATPLite luminescent assay.

Notes: All group values are given as mean \pm SD. $* P<0.05$, $* * P<0.01$ and $* * * P<0.001$.

Abbreviation: NAC, $\mathrm{N}$-acetylcysteine.

of PKM, Glut1, HK2, LDHA, Eno1, PGK1 and GAPDH and protein expression of Glut1 were significantly increased in the BCPAP-SIRT6 group compared with the negative control. Meanwhile, we further found that glucose uptake, lactate production and ATP content in the BCPAP-SIRT6 group were also increased. Meanwhile, these measurements in BCPAP-shSIRT6 showed significant downregulation. Changes both in gene and cellular levels fully proved that SIRT6 promoted the Warburg effect in PTC cells.

Furthermore, transcription factors play adirect role in regulation of these key genes by binding to gene promoters. Previous studies about SIRT6 and the Warburg effect also focused on transcription factors: HIF- $1 \alpha$ and c-MYC. ${ }^{5,6}$ In these studies, SIRT6 inhibited the Warburg effect via taking arole as arepressor of HIF-1 $\alpha$ or cMYC. However, in our study, SIRT6 showed promotion of the Warburg effect. Therefore, we further detected two common transcription factors that contributed to the Warburg effect: c-MYC and p53. As was expected, no expression changes of c-MYC or p53 between the BCPAP-SIRT6 and BCPAP-NC groups were found. These results indicated that the Warburg effect influenced by SIRT6 may not be associated with transcription factors. Multiple studies have demonstrated that ROS could play arole as aWarburg effect stimulant. ${ }^{9}$ Amice model lacking another sirtuin family member, SIRT3, exhibited abnormally high levels of ROS and promoted the Warburg effect. ${ }^{1010}$ Interestingly, in hepatocellular carcinoma cells and oxidative stress-induced neuronal damage, overexpression of SIRT6 increased ROS production. Therefore, we speculated that SIRT6 promotes the Warburg effect by increasing ROS. As expected, ROS production performed with DCFH-DA showed significant increment in the BCPAP-SIRT6 group compared with the BCPAP-NC group. Furthermore, after inhibition of ROS in the BCPAPSIRT6 group with NAC, the mRNA expression of PKM, Glut1, HK2, LDHA, Eno1, PGK1, and GAPDH and protein expression of Glut1 were all significantly rescued. Meanwhile, the increased glucose uptake, lactate production and ATP content of BCPAP-SIRT6 also fell back to the original level.

\section{Conclusion}

From all we have discussed above, we can safely draw the conclusion that SIRT6 promotes the Warburg effect in PTC cells via increasing ROS production. SIRT6 and its upregulated ROS level as well as Warburg effect genes may work as atherapeutic target for PTC.

\section{Ethics approval and consent to participate}

Not applicable.

\section{Consent for publication}

Not applicable.

\section{Availability of data and material}

The datasets used and analyzed during the current study are available from the corresponding author on reasonable request. 


\section{Acknowledgments}

This study was funded by Specialty Feature Construction Project of Pudong Health and Family Planning Commission of Shanghai (Grant No. PWZzb2017-21) and Academic Leaders Training Program of Pudong Health Bureau of Shanghai (Grant No. PWRd2014-07)

\section{Author contributions}

Zhou Yang and Weiping Yu equally implemented the experiment. Renhong Huang contributed to data statistics. Min Ye and Zhijun Min contributed to research design. All authors contributed to data analysis, drafting and revising the article, gave final approval of the version to be published, and agree to be accountable for all aspects of the work.

\section{Disclosure}

The author reports no conflicts of interest in this work.

\section{References}

1. BaiL, LinG, SunL, etal. Upregulation of SIRT6 predicts poor prognosis and promotes metastasis of non-small cell lung cancer via the ERK1/2/MMP9 pathway. Oncotarget. 2016;7(26):40377-40386. doi:10.18632/oncotarget.9750
2. ZhangJ, YinXJ, XuCJ, etal. The histone deacetylase SIRT6 inhibits ovarian cancer cell proliferation via down-regulation of Notch 3 expression. Eur Rev Med Pharmacol Sci. 2015;19(5):818-824.

3. QuN, HuJQ, LiuL, etal. SIRT6 is upregulated and associated with cancer aggressiveness in papillary thyroid cancer via BRAF/ERK/ Mcl1 pathway. IntJOncol. 2017;50(5):1683-1692. doi:10.3892/ ijo.2017.3951

4. LibertiMV, LocasaleJW. The Warburg effect: how does it benefit cancer cells? Trends in Biochemical Sciences. 2016;41(3):211-218. doi:10.1016/j.tibs.2015.12.001

5. ZhongL, D'UrsoA, ToiberD, etal. The histone deacetylase Sirt6 regulates glucose homeostasis via Hiflalpha. Cell. 2010;140(2):280293. doi:10.1016/j.cell.2009.12.041

6. SebastianC, ZwaansBM, SilbermanDM, etal. The histone deacetylase SIRT6 is atumor suppressor that controls cancer metabolism. Cell. 2012;151(6):1185-1199. doi:10.1016/j.cell.2012.10.047

7. CoelhoRG, CazarinJM, Cavalcanti de AlbuquerqueJP, de AndradeBM, CarvalhoDP. Differential glycolytic profile and Warburg effect in papillary thyroid carcinoma cell lines. Oncol Rep. 2016;36(6):3673-3681. doi:10.3892/or.2016.5142

8. ChaiYJ, YiJW, OhSW, etal. Upregulation of SLC2 (GLUT) family genes is related to poor survival outcomes in papillary thyroid carcinoma: analysis of data from the cancer genome atlas. Surgery. 2017;161(1):188-194. doi:10.1016/j.surg.2016.04.050

9. HartPC, MaoM, AbreuALPD, etal. MnSOD upregulation sustains the Warburg effect via mitochondrial ROS and AMPK-dependent signalling in cancer. Nature Communications. 2015;6(6):6053. doi:10.1038/ ncomms 7053

10. BellEL, EmerlingBM, RicoultSJH, GuarenteL. SirT3 suppresses hypoxia inducible factor $1 \alpha$ and tumor growth by inhibiting mitochondrial ROS production. Oncogene. 2011;30(26):2986-2996. doi:10.1038/onc.2011.37
OncoTargets and Therapy

\section{Publish your work in this journal}

OncoTargets and Therapy is an international, peer-reviewed, open access journal focusing on the pathological basis of all cancers, potential targets for therapy and treatment protocols employed to improve the management of cancer patients. The journal also focuses on the impact of management programs and new therapeutic

\section{Dovepress}

agents and protocols on patient perspectives such as quality of life, adherence and satisfaction. The manuscript management system is completely online and includes a very quick and fair peer-review system, which is all easy to use. Visit http://www.dovepress.com/ testimonials.php to read real quotes from published authors. 